\title{
Imprints of the nuclear symmetry energy on the tidal deformability of neutron stars
}

\author{
Plamen G. Krastev ${ }^{1}$ and Bao-An $\mathrm{Li}^{2}$ \\ ${ }^{1}$ Harvard University, Faculty of Arts and Sciences, Research Computing, 38 Oxford \\ Street, Cambridge, MA 02138, U.S.A. \\ 2 Department of Physics and Astronomy, Texas A\&M University-Commerce, P.O. \\ Box 3011, Commerce, TX 75429, U.S.A. \\ E-mail: plamenkrastev@fas.harvard.edu, Bao-An.Li@tamuc.edu
}

\begin{abstract}
Applying an equation of state (EOS) with its symmetric nuclear matter (SNM) contribution and low-density symmetry energy $E_{\text {sym }}(\rho)$ constrained by heavyion reaction data, we calculate the dimensionless tidal deformability $\Lambda$ of neutron stars in coalescing binary systems. Corresponding to the partially constrained EOS that previously predicted a radius of $11.5 \mathrm{~km} \leq R_{1.4} \leq 13.6 \mathrm{~km}$ for canonical neutronstar configurations, $\Lambda$ is found to be in the range of $292 \leq \Lambda_{1.4} \leq 680$, consistent with the very recent observation of the GW170817 event. We investigate the effect of the high-density behavior of $E_{\text {sym }}(\rho)$ on the tidal properties of neutron stars and find that while $\Lambda$ depends strongly on the details of the symmetry energy, different trends of $E_{\text {sym }}(\rho)$ lead to very similar values of $\Lambda$. In particular, the transition from stiff/soft to soft/stiff $E_{\text {sym }}(\rho)$ could yield the same $\Lambda$. Thus, measuring $\Lambda$ alone may not determine completely the density dependence of the symmetry energy. Coherent analyses of the dense neutron-rich nuclear matter EOS underlying both nuclear laboratory experiments and astrophysical observations are therefore necessary to break this degeneracy and determine precisely the details of the $E_{\text {sym }}(\rho)$.
\end{abstract}

Keywords: neutron stars, gravitational waves, equation of state of dense matter

Submitted to: J. Phys. G: Nucl. Part. Phys.

\section{Introduction}

The very first gravitational wave detection, GW170817, from a binary neutron-star merger [1, 2, 3], together with its electromagnetic (EM) counterpart, AT2017gfo (see, e.g., Ref. [4] and references therein), marks the beginning of the new era of multimessenger astronomy, and already started to provide important insights for astrophysics, cosmology, production of heavy elements, and nature of dense matter. Because gravity interacts extremely weakly with matter, gravitational waves (GWs) could deliver detailed information about the neutron star structure and the underlying EOS of dense matter that is unaccessible with conventional astronomical observations. The connection between the gravitational wave signal and the neutron star EOS is provided by the tidal distortion of the star in the extremely strong gravitational field of its companion during 
the inspiral [5]. Although the influence of the EOS on the waveform is most significant during the later stages of the inspiral and merger of two neutron stars, it has been pointed out [6] that tidal deformation effects could be potentially measurable at earlier times of the binary dynamics when the gravitational wave signal is relatively clean. These effects are quantified in terms of a single parameter - the tidal deformability, $\lambda$, which characterizes the quadruple deformation of the star in the tidal gravitational field of its companion (see, e.g., Ref. [7]).

While earlier studies have shown that tidal effects modify the waveform only at the end of an inspiral, and their signature may not be distinguishable from its pointparticle post Newtonian shape [5, 8, 9, 10], the joint detection of the GW170817 signal by the LIGO and Virgo observatories has demonstrated clearly the measurability of tidal effects and placed a constraint on the tidal deformability of neutron stars [1]. With the projected sensitivities of later generation ground-based detectors, such as the Advanced LIGO [11, Virgo [12] and the Kamioka Gravitational Wave Detector (KAGRA) [13, 14, it is expected these effects to be observed in details in the near future. In addition, with the rapid advancement of the detection technologies and the planned new generation gravitational wave observatories, it becomes therefore timely and important to provide reliable estimates of the tidal deformation effects expected in inspiraling binary neutron stars. These estimates play an important role in the overall framework for extracting information on the underlying neutron - star EOS, and together with waveform templates constructed with the help of state-of-the-art numerical simulations (see, e.g., Refs. [7, 15]) and novel analytic techniques [16], could provide valuable guidance for both the detection and interpretation of the gravitational wave signals from current and future detectors.

The response of a neutron star to an applied tidal gravitational field is quantified by the tidal deformability $\lambda$, which depends on the details of the EOS of dense neutron-rich matter. In cold neutron star matter, the nucleonic part of the EOS can be written in terms of the energy per nucleon as

$$
E_{n}(\rho, \delta) \approx E_{0}(\rho)+E_{\mathrm{sym}}(\rho) \delta^{2}
$$

where $E_{0}(\rho)=E_{n}(\rho, 0)$ is the energy per nucleon of symmetric nuclear matter (SNM), $E_{\text {sym }}(\rho)$ is the symmetry energy, and $\delta=\left(\rho_{\mathrm{n}}-\rho_{\mathrm{p}}\right) / \rho$ is the isospin asymmetry, with $\rho_{\mathrm{n}}, \rho_{\mathrm{p}}$ and $\rho=\rho_{\mathrm{n}}+\rho_{\mathrm{p}}$, the neutron, proton and total density respectively. Expanding $E_{0}(\rho)$ up to third order and $E_{\text {sym }}(\rho)$ up to second order around the saturation density $\rho_{0}$ we obtain:

$$
E_{0}(\rho) \approx E_{0}\left(\rho_{0}\right)+\frac{K_{0}}{2}\left(\frac{\rho-\rho_{0}}{3 \rho_{0}}\right)^{2}+\frac{J_{0}}{6}\left(\frac{\rho-\rho_{0}}{3 \rho_{0}}\right)^{3}
$$

and

$$
E_{\text {sym }}(\rho) \approx E_{\text {sym }}\left(\rho_{0}\right)+L\left(\frac{\rho-\rho_{0}}{3 \rho_{0}}\right)+\frac{K_{\text {sym }}}{2}\left(\frac{\rho-\rho_{0}}{3 \rho_{0}}\right)^{2} .
$$

The incompressibility $K_{0}$, the skewness coefficient $J_{0}$, the symmetry energy slope $L$, and the symmetry energy curvature $K_{\text {sym }}$ evaluated at $\rho_{0}$ are defined in, e.g., Ref. [17]. Presently, the EOS of cold nuclear matter under extreme conditions 
of density, pressure and/or isospin asymmetry still remains rather uncertain and theoretically controversial, in particular at supra - saturation densities. Besides the tight constraint provided by the maximum mass of neutron stars, extensive analyses of experimental data of heavy-ion reactions from intermediate to relativistic energies, especially various forms of nucleon collective flow and the kaon production, have constrained reasonably tightly the EOS of SNM up to about $4.5 \rho_{0}$, see, e.g., Ref. [18]. However, at higher densities the EOS remains largely uncertain mainly due to the poorly known high-density behavior of the nuclear symmetry energy $E_{\text {sym }}(\rho)$, see, e.g., Refs. [19, 20, 21, 22, 23, 24, 25]. Besides astrophysical observations, both nuclear structure and reactions, especially with radioactive beams, provide useful means to probe $E_{\text {sym }}(\rho)$ [26]. Indeed, thanks to the efforts and collaborations of both the nuclear physics and astrophysics communities, significant progress has been made in recent years in constraining the symmetry energy around and below nuclear matter saturation density using results from both astrophysical observations and terrestrial nuclear experiments, see, e.g., Refs. [27, 28, 29, 30, 31, 32]. Previously, several authors have studied neutronstar tidal effects in inspiraling binaries and their detectability using both polytropic [33, 34, 35] and hadronic [5, 36, 37, 38, equations of state, and investigated the influence of the EOS on the waveform. The GW170817 event has renewed the interest in the EOS of dense neutron-rich matter and propelled an enormous amount of new detailed studies of various aspects of the EOS and its applications to the physics of neutron stars and gravitational waves. Specifically, new constraints on the neutron-star radius and the EOS of dense matter have been reported based on the extracted tidal deformability from the GW170817 observation. For instance, the LIGO and Virgo collaborations have recently refined their analysis [3] and have reported the radii of the neutron-star binary components in the range of $10.5 \mathrm{~km} \leq R_{\{1,2\}} \leq 13.3 \mathrm{~km}$. Other studies, based on various many-body methods and nuclear interactions, have inferred a rather consistent upper limit on the canonical neutron-star radius of $R_{1.4} \leq 13.7 \mathrm{~km}$ [39, 40, 41, 42, 43, 44, 45, 46] using the original findings of Ref. [1].

In this work, applying an EOS with its SNM part and low-density $E_{\text {sym }}(\rho)$ constrained by heavy-ion reaction data, we calculate the tidal deformability of neutron stars in coalescing binary systems and examine the effects of $E_{\text {sym }}(\rho)$ on $\lambda$. In particular, we investigate the impact of transition from stiff/soft to soft/stiff symmetry energy on the tidal properties of neutron stars and discuss the implications of our results for the EOS of dense matter and the interpretation of current and future gravitation-wave signals from inspiraling neutron-star binaries.

This paper is organized as follows. After the introductory remarks in this section, in Sec. 2 we discuss the necessary formalism to calculate the tidal deformability $\lambda$. In Sec. 3 we briefly review the main features of the partially constrained EOS. We present our results for the tidal deformability of neutron stars and discuss the effects of the EOS in Sec. 4. At the end, we conclude in Sec. 5 with a short summary and outlook of future investigations.

Conventions: We use units in which $G=c=1$. 


\section{Formalism for calculating the neutron star tidal deformability}

In this section we briefly recall the formalism for calculating the neutron star tidal deformability $\lambda$. As two neutron stars approach each other during the early stages of an inspiral they experience tidal deformation effects quantified in terms of $\lambda$. This parameter is defined as [5, 6, 35]

$$
\lambda=-\frac{Q_{i j}}{\mathcal{E}_{i j}}
$$

where $Q_{i j}$ is the induced mass quadruple moment of a star in the gravitational tidal field $\mathcal{E}_{i j}$ of its companion. The tidal deformability can be expressed in terms of the neutron star radius, $R$, and dimensionless tidal Love number, $k_{2}$ as

$$
\lambda=\frac{2}{3} k_{2} R^{5}
$$

The tidal Love number $k_{2}$ is calculated using the following expression [33, 36, 37]

$$
\begin{aligned}
k_{2}\left(\beta, y_{R}\right) & =\frac{8}{5} \beta^{5}(1-2 \beta)^{2}\left[2-y_{R}+2 \beta\left(y_{R}-1\right)\right] \\
& \times\left\{2 \beta\left[6-3 y_{R}+3 \beta\left(5 y_{R}-8\right)\right]\right. \\
& +4 \beta^{3}\left[13-11 y_{R}+\beta\left(3 y_{R}-2\right)\right. \\
& \left.+2 \beta^{2}\left(1+y_{R}\right)\right]+3(1-2 \beta)^{2}\left[2-y_{R}\right. \\
& \left.\left.+2 \beta\left(y_{R}-1\right)\right] \ln (1-2 \beta)\right\}^{-1},
\end{aligned}
$$

where $\beta \equiv M / R$ is the dimensionless compactness parameter and $y_{R} \equiv y(R)$ is solution of the following first order differential equation

$$
\frac{d y(r)}{d r}=-\frac{y(r)^{2}}{r}-\frac{y(r)}{r} F(r)-r Q(r),
$$

with

$$
\begin{aligned}
F(r) & =\left\{1-4 \pi r^{2}[\varepsilon(r)-p(r)]\right\}\left[1-\frac{2 m(r)}{r}\right]^{-1}, \\
Q(r) & =4 \pi\left[5 \varepsilon(r)+9 p(r)+\frac{\varepsilon(r)+p(r)}{c_{s}^{2}(r)}-\frac{6}{r^{2}}\right]\left[1-\frac{2 m(r)}{r}\right]^{-1} \\
& -\frac{4 m^{2}(r)}{r^{4}}\left[1+\frac{4 \pi r^{3} p(r)}{m(r)}\right]^{2}\left[1-\frac{2 m(r)}{r}\right]^{-2},
\end{aligned}
$$

where $c_{s}^{2}(r) \equiv d p(r) / d \varepsilon(r)$ is the squared speed of sound. Starting at the center of the star, for a given EOS Eq. (77) needs to be integrated self-consistently together with the Tolman-Oppenheimer-Volkoff equations, i.e.,

$$
\begin{aligned}
& \frac{d p(r)}{d r}=-\frac{\varepsilon(r) m(r)}{r^{2}}\left[1+\frac{p(r)}{\varepsilon(r)}\right]\left[1+\frac{4 \pi r^{3} p(r)}{m(r)}\right]\left[1-\frac{2 m(r)}{r}\right]^{-1}, \\
& \frac{d m(r)}{d r}=4 \pi \varepsilon(r) r^{2} .
\end{aligned}
$$

Imposing the boundary conditions at $r=0$ such that, $y(0)=2, m(0)=0$, and $p(0)=p_{c}$, the Love number $k_{2}$ and the tidal deformability $\lambda$ can be readily calculated. One can also 
compute the dimensionless tidal deformability $\Lambda$, which is related to the compactness parameter $\beta$ and the Love number $k_{2}$ through

$$
\Lambda=\frac{2}{3} \frac{k_{2}}{\beta^{5}}
$$

The total tidal effect of two neutron stars in an inspiraling binary system is given by the mass-weighted (dimensionless) tidal deformability (see, e.g., Refs. [5, 35])

$$
\tilde{\Lambda}=\frac{16}{13} \frac{\left(M_{1}+12 M_{2}\right) M_{1}^{4} \Lambda_{1}+\left(M_{2}+12 M_{1}\right) M_{2}^{4} \Lambda_{2}}{\left(M_{1}+M_{2}\right)^{5}},
$$

where $\Lambda_{1}=\Lambda_{1}\left(M_{1}\right)$ and $\Lambda_{2}=\Lambda_{2}\left(M_{2}\right)$ are the (dimensionless) tidal deformabilities of the individual binary components. As pointed out previously [5], although $\Lambda$ is calculated for single neutron stars, the universality of the neutron-star EOS allows us to predict the tidal phase contribution for a given binary system from each EOS. For equal-mass binary systems $\tilde{\Lambda}$ reduces to $\Lambda$. The weighted (dimensionless) deformability $\tilde{\Lambda}$ is usually plotted as a function of the chirp mass $\mathcal{M}=\left(M_{1} M_{2}\right)^{3 / 5} / M_{T}^{1 / 5}$ for various values of the asymmetric mass ratio $\eta=M_{1} M_{2} / M_{T}^{2}$, where $M_{T}=M_{1}+M_{2}$ is the total mass of the binary.

\section{Partially constrained equation of state of neutron-rich matter with the MDI (momentum-dependent interaction)}

The tidal deformability depends on the neutron-star EOS through both the tidal Love number $k_{2}$ and stellar radius $R$ (see Eq. (5) ). As already mentioned in the introduction, current theoretical predictions of the nucleonic EOS diverge widely mainly because of the uncertain density dependence of the nuclear symmetry energy, especially at high densities. In the ongoing efforts to constrain the EOS and provide useful guidance to theoretical models, it is very important and timely to determine what information could be extracted from precise measurements of $\Lambda$, and also what aspects of the EOS could be better determined with the help of this information. This is clearly a twofold problem. On the one hand, gravitational wave observations from inspiraling compact binaries are expected to place more stringent constraints on the EOS, in particular on the highdensity behavior of the symmetry energy (see, e.g., Ref. [47]). On the other hand, to extract useful information on the details of the EOS from gravitational waves, one needs reliable estimates of the tidal deformation effects and accurate waveform templates, which require reliable estimates of $\Lambda$. To provide accurate estimates of the tidal deformability one needs to reduce the uncertainty of the high-density $E_{\text {sym }}(\rho)$. Because the MDI EOS [48, 49] has its SNM part and symmetry energy $E_{\text {sym }}(\rho)$ constrained by heavy-ion reaction data up to about $4.5 \rho_{0}$ and $1.2 \rho_{0}$ respectively, it would be interesting to compare the $\Lambda$ values using the MDI EOS with the recent observational constraints from LIGO/Virgo [1, 2, 3]. In this study we assume a simple model of stellar matter of nucleons and light leptons (electrons and muons) in beta-equilibrium.

Below we briefly summarize the most relevant features of the MDI EOS. (For a comprehensive discussion see, e.g., Refs. [48, 49].) With the MDI interaction, the 


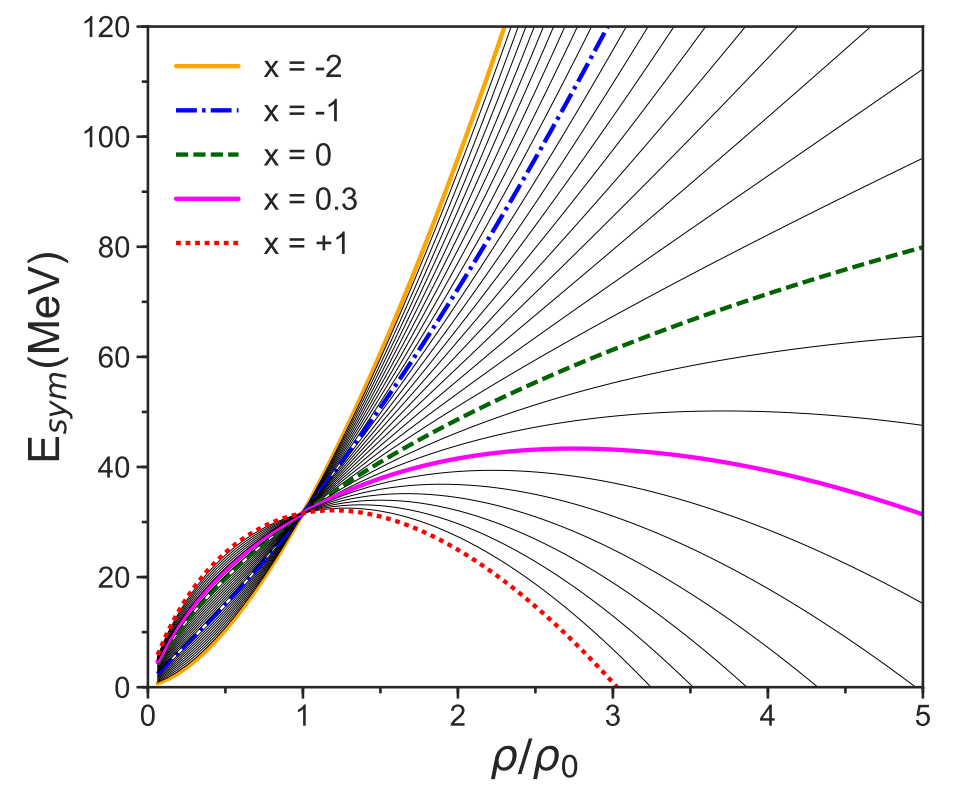

Figure 1. (Color online) The density dependence of nuclear symmetry energy using the MDI interaction by varying the parameter $x$ which measures the relative strength of the effective three-body force in the isotriplet channel with respect to the isosinglet channel. In the figure $x$ is varied from -2 to +1 in steps of $\Delta x=0.1$.

potential energy density $V(\rho, T, \delta)$ of an asymmetric nuclear matter at finite temperature $T$ is parameterized as [48, 50]

$$
\begin{aligned}
V(\rho, T, \delta) & =\frac{A_{u}(x) \rho_{n} \rho_{p}}{\rho_{0}}+\frac{A_{l}(x)}{2 \rho_{0}}\left(\rho_{n}^{2}+\rho_{p}^{2}\right)+\frac{B}{\sigma+1} \frac{\rho^{\sigma+1}}{\rho_{0}^{\sigma}} \\
& \times\left(1-x \delta^{2}\right)+\frac{1}{\rho_{0}} \sum_{\tau, \tau^{\prime}} C_{\tau, \tau^{\prime}} \\
& \times \iint d^{3} p d^{3} p^{\prime} \frac{f_{\tau}(\vec{r}, \vec{p}, T) f_{\tau^{\prime}}\left(\vec{r}, \vec{p}^{\prime}, T\right)}{1+\left(\vec{p}-\vec{p}^{\prime}\right)^{2} / \Lambda^{2}},
\end{aligned}
$$

where $f_{\tau}(\vec{r}, \vec{p}, T)$ is the nucleon phase-space distribution function at coordinate $\vec{r}$, momentum $\vec{p}$ and temperature $T$. The corresponding single-particle potential deduced from the Hartree-Fock approach is given by

$$
\begin{aligned}
& U_{\tau}(\rho, T, \delta, \vec{p})=A_{u}(x) \frac{\rho_{-\tau}}{\rho_{0}}+A_{l}(x) \frac{\rho_{\tau}}{\rho_{0}} \\
& +B\left(\frac{\rho}{\rho_{0}}\right)^{\sigma}\left(1-x \delta^{2}\right)-8 \tau x \frac{B}{\sigma+1} \frac{\rho^{\sigma-1}}{\rho_{0}^{\sigma}} \delta \rho_{-\tau} \\
& +\sum_{t=\tau,-\tau} \frac{2 C_{\tau, t}}{\rho_{0}} \int d^{3} \vec{p}^{\prime} \frac{f_{t}\left(\vec{r}, \vec{p}^{\prime}, T\right)}{1+\left(\vec{p}-\vec{p}^{\prime}\right)^{2} / \Lambda^{2}},
\end{aligned}
$$

where $\tau=1 / 2(-1 / 2)$ for neutrons (protons). The parameters $x, A_{u}(x), A_{\ell}(x), B$, $C_{\tau, \tau}, C_{\tau,-\tau}, \sigma$, and $\Lambda$ are fixed by saturation properties of SNM, both isoscalar and isovector nucleon optical potentials, as well as a specified magnitude and slope of the symmetry energy at saturation density of nuclear matter as discussed in detail in Ref. [48]. 


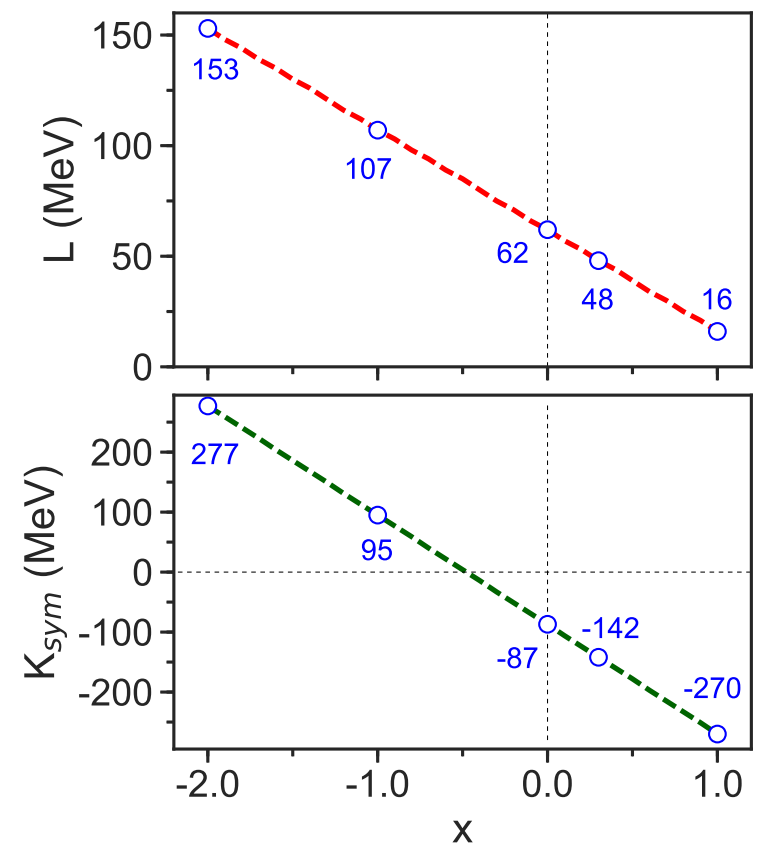

Figure 2. (Color online) Slope of symmetry energy $L$ (upper panel) and curvature of symmetry energy $K_{\text {sym }}$ (lower panel) evaluated at saturation density $\rho_{0}$ as functions of the parameter $x$. Numerical values are shown at $x$ equal to $-2,-1,0,0.3$ and +1 .

The parameter $x$ in Eq. (14) was introduced to account for the uncertain spinisospin dependence of the three-body nuclear force in dense neutron-rich matter. It measures the relative strength of the isotriplet to isosinglet interaction of the effective three-body force in the modified Gogny interaction. By varying the parameter $x$, one can mimic the largely uncertain density dependence of the nuclear symmetry energy $E_{\text {sym }}(\rho)$ as predicted by different nuclear many-body theories using various interactions. By design, different values of $x$ can lead to widely different trends for the $E_{\text {sym }}(\rho)$ without changing the SNM EOS and the magnitude of the symmetry energy at saturation density. This is illustrated in Fig. 1 where we show representative examples of the $E_{\text {sym }}(\rho)$ for values of $x$ in the interval between -2 and +1 . Here we need to emphasize that various values of $x$ correspond to various values of $L$ and $K_{\text {sym }}$, i.e., varying $x$ changes both parameters simultaneously as shown in Fig. 2.

The MDI interaction has been used extensively in studies of heavy-ion reactions and neutron stars. It has also been used to investigate thermodynamical properties of hot, dense and neutron-rich matter, see, e.g., Refs. [51, 52]. Previously it has been demonstrated that only EOSs with values of $x$ in the range between -1 and 0 have symmetry energy consistent with the terrestrial nuclear laboratory data [50, 53, 54]. Therefore these two limiting cases are expected to determine the boundaries of the most probable neutron star configurations, and in turn the range of the most probable values of the (dimensionless) tidal deformability $\Lambda$. For the purpose of this study, we consider EOSs with several representative values of $x$ : $-2,-1,0$, and 0.3 .

We show the EOSs applied in this work in Fig. 3. The upper panel displays the total energy density, $\varepsilon$, as a function of the baryon number density, and the lower panel 


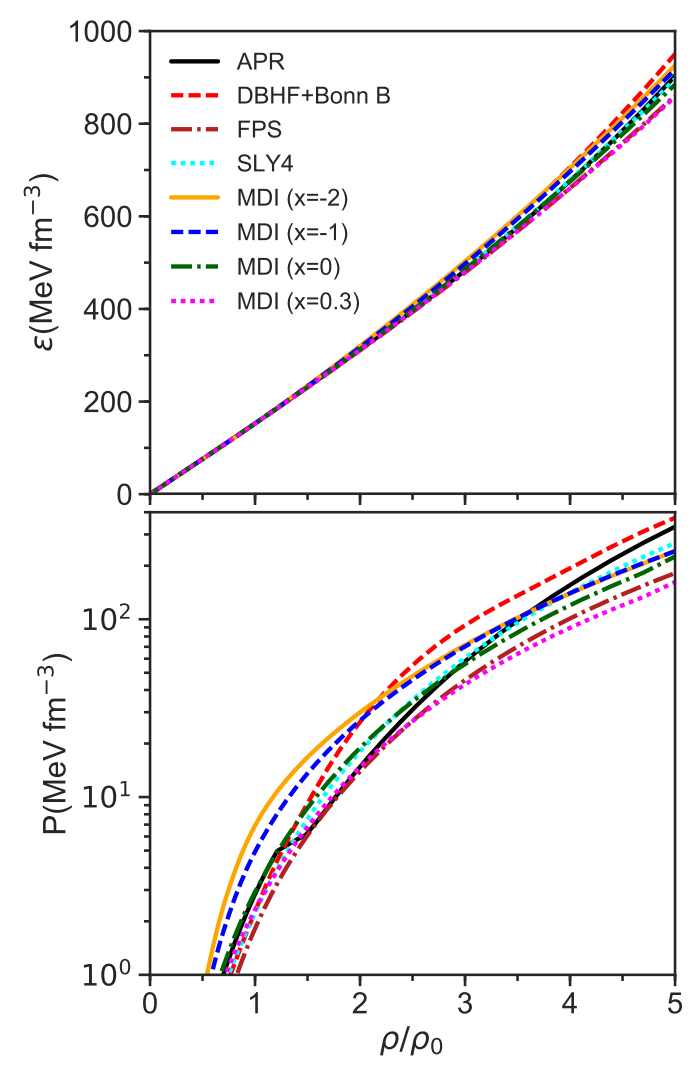

Figure 3. (Color online) Equation of state. The upper frame shows the mass energy density as a function of baryon density (in units of saturation density, $\rho_{0} \approx 0.16 \mathrm{fm}^{-3}$ ) and the lower frame shows the total pressure (including the lepton contributions) versus baryon density. (The "dip" exhibited by the density curve of the APR EOS is due to a phase transition from low density phase (LDP) to high density phase (HDP). See Akmal et al. [55] for details.)

shows the total pressure $P$. In addition to the MDI EOS, we also include several EOSs frequently used in studies of neutron-star properties and related phenomena. Namely, we also display results by Akmal et al. [55] with the $A 18+\delta v+U I X *$ interaction (APR), Dirac-Brueckner-Hartree-Fock (DBHF) calculations [56, 57] with Bonn B One-BosonExchange (OBE) potential [58] (DBHF+Bonn B), Pandharipande and Ravenhall [59] (FPS), and Douchin and Haensel [60] (SLY4). Below approximately $0.07 \mathrm{fm}^{-3}$ the EOSs shown in Fig. 3 are supplemented by a crustal EOS, which is more suitable at lower densities. For the purpose of this work we assume the same core-to-crust transition density for all MDI EOSs with various values of $x$. For the inner crust we apply the EOS by Pethick et al. 61] and for the outer crust the one by Haensel and Pichon [62]. At higher densities we assume a continuous functional for the equations of state.

We emphasize that the EOS of SNM with the MDI interaction is constrained by the available data on collective flow and kaon production in relativistic heavy-ion collisions [18] (see, e.g., Fig. 1 in Ref. [63]), while the $E_{\text {sym }}(\rho)$ is constrained around the saturation density by isospin diffusion data [53] to be between that with $x=-1$ and $x=0$ [50, 54]. Such a MDI EOS constrained by the terrestrial laboratory data has been used in various calculations of neutron star properties and astrophysical phenomena 


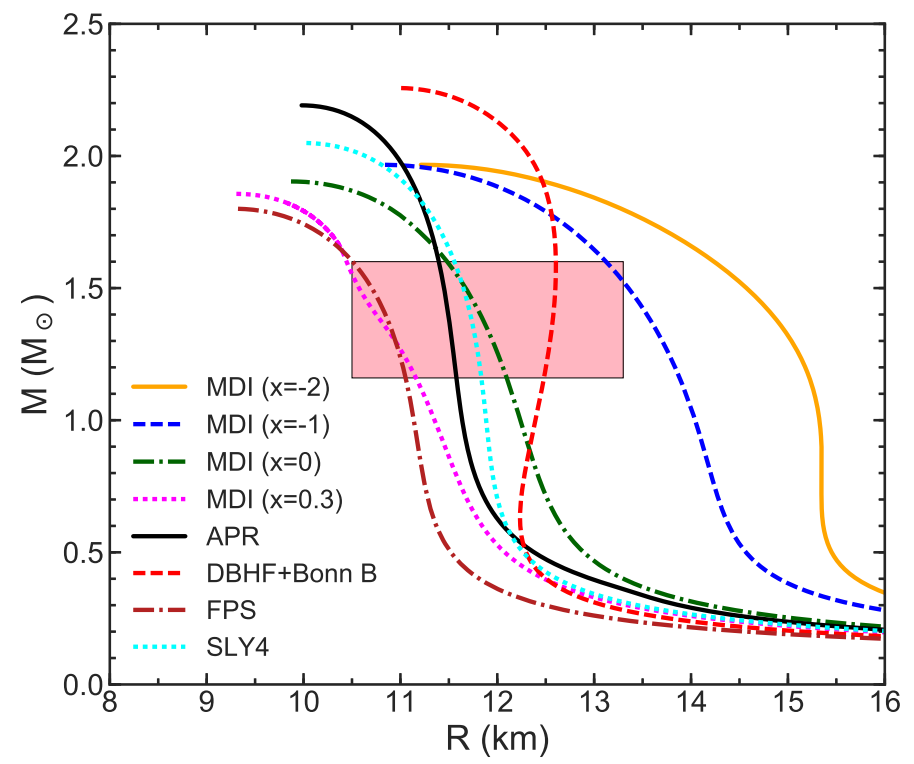

Figure 4. (Color online) Neutron star mass-radius relation for the EOSs applied in this study. The shaded region enclosed between 1.16 and $1.6 M_{\odot}$ and 10.5 and 13.3 $\mathrm{km}$ corresponds to the constraints on the neutron-star radius from the refined analysis of the GW170817 observation [3].

since about 2006. For example, it has been used to constrain the neutron star radius [54] with an estimated range consistent with the observational data. More quantitatively, corresponding to the parameter $x$ between 0 and -1, the MDI EOS predicted a radius of $11.5 \mathrm{~km}<R_{1.4}<13.6 \mathrm{~km}$ for a canonical neutron star of mass $1.4 M_{\odot}$ 54. This result was later found consistent with estimates using the spin rate of the fastest pulsar PSRJ1748-2446ad [64], thermonuclear bursts on neutron star surfaces and spectra of neutron stars in quiescence [31]. Interestingly, the limits of $10.5 \mathrm{~km} \leq R_{\{1,2\}} \leq 13.3 \mathrm{~km}$ at the $90 \%$ credible level, for the component masses between 1.16 and $1.60 M_{\odot}$, determined very recently by the refined analysis of the the GW170817 observation [3] are consistent with but less restrictive than the existing constraints on the radii of canonical neutron stars mentioned above. Since the correlation between the radii of neutron stars and sizes of neutron-skins of heavy nuclei has been an interesting topic in exploring the EOS of neutron-rich matter using the multi-messenger approach [65, it is worth noting that the size of neutron-skin in ${ }^{208} \mathrm{~Pb}$ was predicted to be $0.22 \mathrm{fm}$ and $0.28 \mathrm{fm}$ with the MDI EOS of $x=0$ and $x=-1$, respectively [54]. The constrained MDI EOS was also applied to study the possible time variation of the gravitational constant $G$ [66] with the help of the gravitochemical heating approach developed by Jofre et al. [67]. In addition, it was also used to limit a number of other global, transport and thermal properties of both static and rapidly rotating neutron stars [63, 68, 69, 70, 71]. 


\section{Results and discussion}

\subsection{Love number and tidal deformability}

The details of the EOS can affect significantly global properties of neutron stars and tidal interactions in inspiraling binary systems. Fig. 4 shows the neutron star mass-radius relation for the EOSs considered in this study.

The generic behavior of the Love number $k_{2}$ is shown in the upper panel of Fig. 5 as a function of stellar mass. The value of $k_{2}$ increases rapidly with the neutron star mass approaching a maximum for models with masses near $1 M_{\odot}$, and then gradually decreases. We observe that there is a moderate variation in $k_{2}$ between results with different EOSs for fixed neutron star mass. This behavior can be understood in terms of the physical significance of the Love number $k_{2}$ - it measures how easily the bulk of the matter in a neutron star is deformed [5]. This implies that more centrally condensed stellar models have smaller Love numbers, and smaller tidal deformation. On the other hand, for smaller compactness, the softer crust becomes a greater fraction of the star, so the star is more centrally condensed and $k_{2}$ becomes smaller.

Next, we turn our attention to the behavior of the tidal deformability $\lambda$. This parameter is proportional to the quantity that is directly measurable by gravitationalwave observations of inspiraling neutron-star binaries [5], and as such has a direct astrophysical significance. We show the tidal deformability in the lower panel of Fig. 5 as a function of the neutron star mass. Except for very low values of the neutron star mass, for each EOS $\lambda$ follows a trend very similar to that of $k_{2}$. However, because in addition to the Love number, $\lambda$ is also proportional to $R^{5}$ and the applied EOSs result in neutron star configurations with a wide range of radii (see Fig. 4), it experiences much greater variations compared to $k_{2}$. The tidal deformability becomes large for neutron star models with mass near $0.1 M_{\odot}$ because they have large radii. We also observe that $\lambda$ becomes larger for stellar configurations with masses around $0.6-1.0 M_{\odot}$, where it shows greater variations for EOSs that produce models with large radii. Here we recall that the neutron star radius is strongly correlated with the density dependence of nuclear symmetry energy [21]. As a quantitative example, it was demonstrated in Ref. [54] that changing the parameter $x$ from 0 to -2 while keeping the incompressibility fixed at $K_{0}=211 \mathrm{MeV}$ leads to a change in radius from about $12 \mathrm{~km}$ to $15 \mathrm{~km}$ for a canonical neutron star without affecting the maximum neutron star mass $\left(1.9 M_{\odot}\right)$ that can be supported by the MDI EOS. Moreover, the $E_{\text {sym }}(\rho)$ of the MDI EOS with $x=0$ is about the same as that of the APR EOS up to approximately $4 \rho_{0}$. However, the APR EOS has a stiffer incompressibility of $K_{0}=269 \mathrm{MeV}$. By examining the predictions for the mass-radius relation, it was found in Ref. [54], and also shown in Fig. 4 in this work, that the APR EOS leads to about a $16 \%$ higher maximum mass $\left(2.2 M_{\odot}\right)$ but only a $5 \%$ decrease in radius (from $12.0 \mathrm{~km}$ to $11.5 \mathrm{~km}$ ) for a canonical neutron star as compared to the MDI results with $x=0$. More qualitatively, an EOS with stiffer $E_{\text {sym }}(\rho)$, such as the MDI EOS with $x=-1$ and $x=-2$, results in less centrally condensed stellar models, and in turn greater radii. Specifically, the MDI $(x=-2)$ EOS produces neutron star configurations with larger radii than those of 


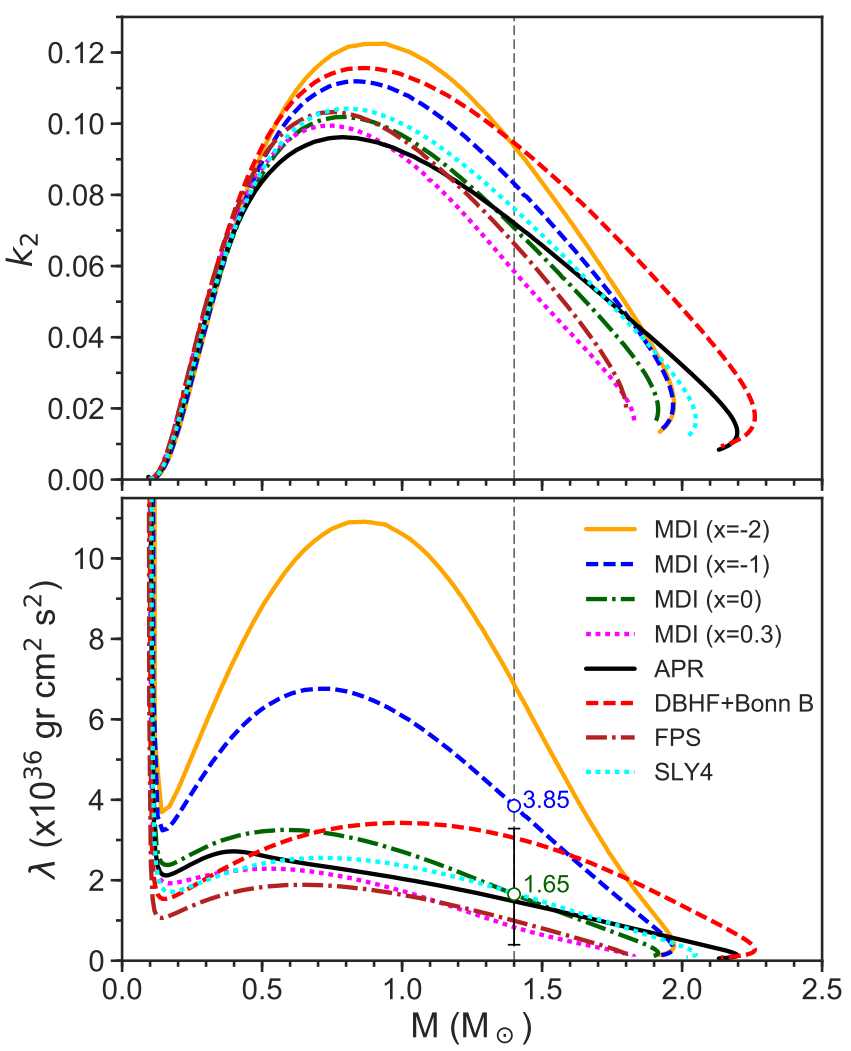

Figure 5. (Color online) Love number (upper panel) and tidal deformability (lower panel) as a function of neutron star mass. The error bar at $1.4 M_{\odot}$ corresponds to the limits on $\lambda_{1.4}$ determined from the refined analysis of GW170817 [3]. The numerical values next to the "o" characters denote $\lambda_{1.4}$, in units of $\left(10^{36} \mathrm{gr} \mathrm{cm}^{2} \mathrm{~s}^{2}\right)$, obtained with the MDI EOS with $x=0$ and $x=-1$.

models from the rest of the EOSs applied here 68. Because $\lambda$ quantifies the neutron star deformation in response to an external tidal field, the results in Fig. 5 suggest that less compact stars are more easily deformed, and more centrally condensed models are more "resistant" to deformation. This is consistent with previous studies which found out that more compact neutron star models are less altered by various deformation driving mechanisms, e.g., rapid rotation [68, 72].

The results in Fig. 5 suggest that $\lambda$ depends on the EOS of stellar matter where the dependence is greater for stellar models with stiffer symmetry energy, and more generally, stiffer EOSs. As already pointed out in previous investigations, e.g., Ref. [47], for neutron stars in the mass-range of interest, $\lambda$ is mostly affected by the highdensity behavior of $E_{\text {sym }}(\rho)$. The symmetry energy has been partially constrained by available terrestrial nuclear laboratory data, in particular around the saturation density. Although at higher densities $E_{\text {sym }}(\rho)$ is presently rather uncertain, these constraints could still provide valuable information about the EOS of dense matter and related neutron star properties. Since the MDI $(x=0)$ and MDI $(x=-1)$ EOSs have symmetry energy consistent with nuclear laboratory data they provide limits on the most probable neutron star configurations, and in turn on the most probable values of $\lambda$, in accord with the available terrestrial nuclear laboratory constraints. In this respect, the region 

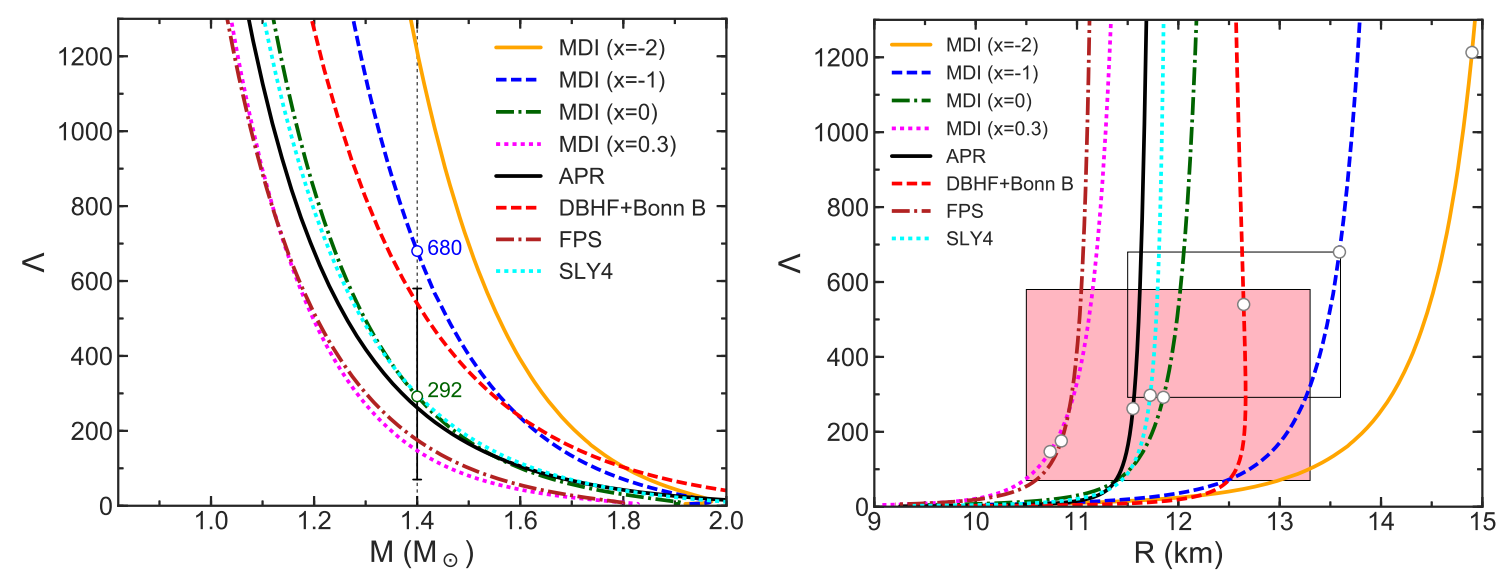

Figure 6. (Color online) Dimensionless tidal deformability as a function of neutronstar mass (left panel) and neutron-star radius (right panel). The error bar at $1.4 M_{\odot}$ in the left window and the colored (light-pink) region of $\Lambda=[70-580]$ and $R=[10.5-13.3] \mathrm{km}$ in the right window indicate the gravitational wave constraints from GW170817 3. Similarly, the constraints based on heavy-ion collisions, obtained with the MDI EOS with $x=0$ and $x=-1$, are denoted by the numerical values next to the " o" characters in the left panel, and the rectangular region of $\Lambda=[292-680]$ and $R=[11.5-13.6] \mathrm{km}$ in the right panel. The dot symbols in the right frame indicate $\Lambda_{1.4}$.

between the $x=0$ and $x=-1$ curves in the lower panel of Fig. 5 denotes the range of the most probable values of the tidal deformability $\lambda$ in equal-mass binaries $(\tilde{\lambda}$ reduces to $\lambda$ in the equal-mass case). The specific numeric values shown in the figure are for neutron star configurations of $1.4 M_{\odot}$. Depending on the details of the EOS, $\lambda$ is found to be in the range of $\sim[1.7-3.9] \times 10^{36}\left(\mathrm{gr} \mathrm{cm}^{2} \mathrm{~s}^{2}\right)$. These estimates, based on nuclear laboratory data, are consistent with the constraints on the tidal deformability by the LIGO/Virgo observation of the GW170817 event [3]. In the case of a low dimensionless spin $(\chi \leq|0.05|)$ of the individual binary components, for $1.4 M_{\odot}$ stellar configurations, Ref. [3] quotes $\Lambda_{1.4}$ in the range of $\sim[70-580]$, or $\lambda \sim[0.4-3.3] \times 10^{36}\left(\mathrm{gr} \mathrm{cm}^{2} \mathrm{~s}^{2}\right)$, where $\lambda=C_{\lambda} \Lambda$ with $C_{\lambda}=G^{4} M^{5} / c^{10}$ (in CGS units). These refined constraints from GW170817 are denoted by the error bar at $1.4 M_{\odot}$ in Fig. 5.

In Fig. 6 we show the dimensionless tidal deformability $\Lambda$ as a function of the neutron star mass (left panel) and stellar radius (right panel). This allows for a direct comparison with the observational constraints from the GW170817 event. Corresponding to the MDI EOS with $x=0$ and $x=-1, \Lambda$ is found to be in the range of $292 \leq \Lambda_{1.4} \leq 680$. The error bar at $1.4 M_{\odot}$ in the left window and the colored rectangular region in the right window represent the tighter constraints from the GW170817 observation [3]. The rectangular region of $\Lambda=[292-680]$ and $R=[11.5-13.6] \mathrm{km}$ represents the constraints on $\Lambda$ and $R$ from heavy ion collision data. As explained in Ref. [54] the minimum radius is extended to $11.5 \mathrm{~km}$ (from 11.9 $\mathrm{km}$ as obtained with the MDI $(x=0)$ EOS $)$ to account for the remaining uncertainty in the symmetric part of the EOS. While both regions, based on the GW170817 analysis and heavy-ion collision data, reasonably overlap, the constraints from nuclear laboratory 
Table 1. Properties of $1.4 M_{\odot}$ neutron star models for the EOSs discussed in the text. The first column identifies the equation of state. The remaining columns exhibit the following quantities: neutron-star radius $R(\mathrm{~km})$; compactness parameter $\beta$; Love number $k_{2}$; dimensionless tidal deformability $\Lambda$; slope of nuclear symmetry energy at saturation density $L(\mathrm{MeV})$.

\begin{tabular}{lccccc}
\hline EOS & $R$ & $\beta$ & $k_{2}$ & $\Lambda$ & $L$ \\
\hline MDI $(x=-2)$ & 14.9 & 0.14 & 0.0936 & 1212 & 153 \\
MDI $(x=-1)$ & 13.6 & 0.15 & 0.0831 & 680 & 107 \\
MDI $(x=0)$ & 11.9 & 0.17 & 0.0707 & 292 & 62 \\
MDI $(x=0.3)$ & 10.7 & 0.19 & 0.0585 & 147 & 48 \\
APR & 11.5 & 0.18 & 0.0721 & 261 & 62 \\
DBHF+Bonn B & 12.6 & 0.16 & 0.0946 & 540 & 69 \\
FPS & 10.8 & 0.19 & 0.0664 & 175 & 35 \\
SLY4 & 11.7 & 0.18 & 0.0762 & 297 & 47 \\
\hline
\end{tabular}

data appear to be more restrictive. For completeness, in Table 1 we list properties of $1.4 M_{\odot}$ neutron-star models calculated with all EOSs applied in this work.

\subsection{Effects of varying the density dependence of $E_{\text {sym }}$ on the tidal deformability}

To investigate further the effects of $E_{\text {sym }}(\rho)$ on $\Lambda$ in the following we study how varying the density dependence of the symmetry energy affects $\Lambda_{1.4}$. In particular, we examine how transitions from stiff to soft and soft to stiff $E_{\text {sym }}(\rho)$ at saturation density $\rho_{0}$ impact the tidal properties of neutron stars. This approach is mainly motivated by the ongoing efforts to pin down the close but not precisely established relation between $\Lambda_{1.4}$ and $R_{1.4}$, and determine what aspects of the EOS of dense neutron-rich matter could be exactly determined by accurate measurements of the tidal deformability. Although some interesting indications and speculations have been examined in recent literature, e.g., correlation between $\Lambda_{1.4}$ and the slope of the symmetry energy $L_{0}$ around $\rho_{0}$, sizes of neutron-skins in heavy nuclei, and/or possible phase transitions in dense neutronrich matter [39, 41, 73, 74, 75, much more work is still required to determine what aspects of the EOS could be revealed from $\Lambda_{1.4}$. Most notably, the fact that latest measurements of neutron star radii from low-mass X-ray binaries favor softer $E_{\text {sym }}(\rho)$ (and EOS) [76, 77, while the measured skin thickness of ${ }^{208} \mathrm{~Pb}$ by the PREX experiment [78, 79] (if confirmed by PREX-II) suggests stiffer $E_{\text {sym }}$ at nuclear densities, led some studies to point out that the evolution from soft to stiff $E_{\text {sym }}$ may be indicating a phase transition in the neutron star interior just above saturation density [41].

In the MDI model the density dependence of the symmetry energy is controlled by the parameter $x$ and, as illustrated in Fig. 1, different values of $x$ lead to a wide range of possible trends for $E_{\text {sym }}(\rho)$. For the purpose of this investigation, we model the transition from one trend of the density dependence of $E_{\text {sym }}$ to another by using different values of $x$ below and above $\rho_{0}$. Namely, for each of several representative 
Imprints of the nuclear symmetry energy on the tidal deformability of neutron stars 14
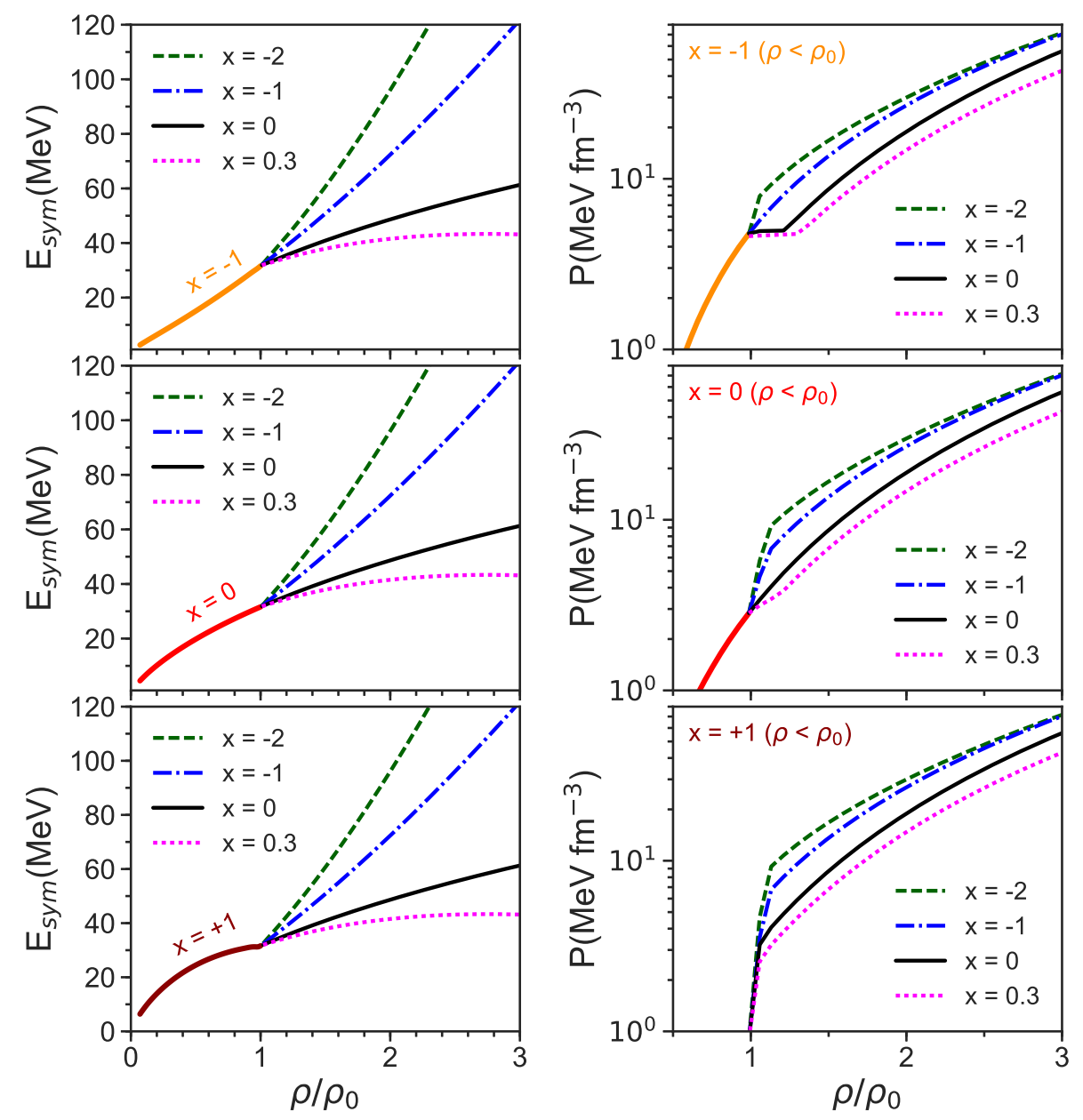

Figure 7. (Color online) Varied symmetry energy (left panel) and pressure (right panel). $E_{\text {sym }}(\rho)$ and $P$ are shown for three representative values of $x$ below saturation density $\rho_{0}$ : -1 (upper windows), 0 (middle windows), and +1 (lower windows). Above $\rho_{0} x$ is varied from -2 to 0.3 . Specifically, for $\rho>\rho_{0}$ we consider $x=[-2,-1,0,0.3]$. See text for details.

values of $x$ below the saturation density, $x=[-1,0,+1]$, we vary $x$ above $\rho_{0}$ from -2 to 0.3 and compute $\Lambda$ with the resultant EOSs. We refer to $E_{\text {sym }}$ with $x=-2$ and -1 as stiff and $E_{\text {sym }}$ with $x=[0,0.3,+1]$ as soft. Or more qualitatively, for the purpose of our analysis we define $E_{\text {sym }}(\rho)$ as stiff for $K_{\text {sym }}>0$ and soft for $K_{\text {sym }}<0$, where as illustrated in Fig. 2, with increasing $x, K_{\text {sym }}$ changes sign at approximately $x=-0.5$. Both branches of $E_{\text {sym }}$, below and above saturation density, are joined as smoothly as possible at $\rho_{0}$. The modified $E_{\text {sym }}(\rho)$ and the resultant total pressure $P$ for these cases are shown in Fig. 7. For instance, in this respect changing $x$ from -1 for $\rho<\rho_{0}$ to 0 or 0.3 for $\rho>\rho_{0}$ represents a transition from stiff to soft symmetry energy (upper panels of Fig. 7). Conversely, changing $x$ from +1 for $\rho<\rho_{0}$ to -1 , or -2 , for $\rho>\rho_{0}$ represents a transition from soft to stiff symmetry energy (lower panels of Fig. 7). Here we recall that both $L$ and $K_{\text {sym }}$ change simultaneously with $x$ (see Fig. 2). Keeping $x$ fixed below $\rho_{0}$ and varying it above the $\rho_{0}$ is effectively equivalent to keeping either $L$ or $K_{\text {sym }}$ fixed and varying the other. A systematic study of the effects of varying 

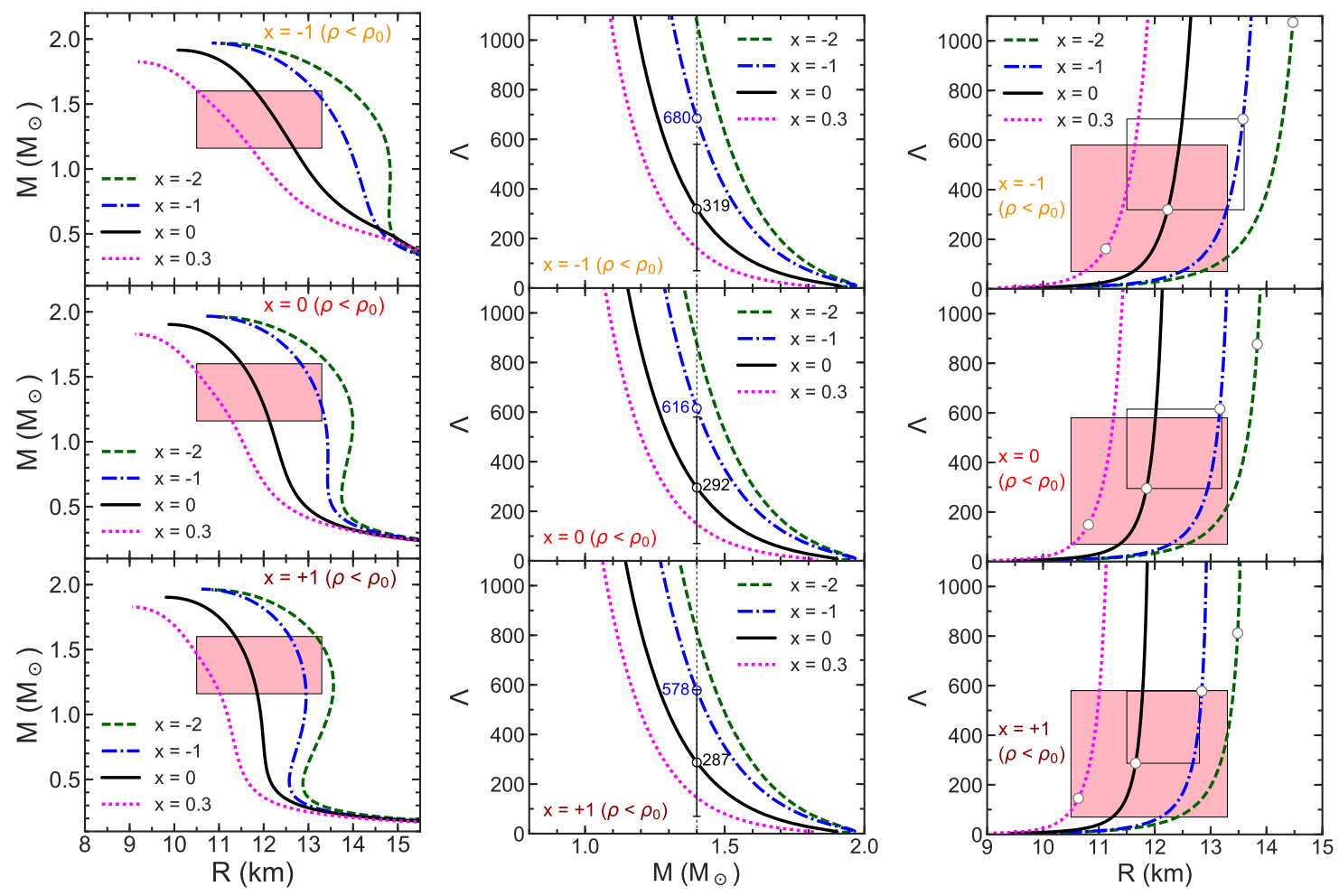

Figure 8. (Color online) Mass-radius relation (left panel) and dimensionless tidal deformability $\Lambda$ as a function of neutron-star mass $M$ (middle panel) and radius $R$ (right panel). In all frames, the curve labels indicate $x$ values above saturation density $\left(x_{\left(\rho>\rho_{0}\right)}\right)$. Colored regions (left and right windows) and the error bar at $1.4 M_{\odot}$ (middle window) represent the gravitational-weave constraints from GW170817 [3]. Uncolored rectangular regions in the right panel indicate $R_{1.4}$ and $\Lambda_{1.4}$ computed with the MDI EOS with $x_{\left(\rho>\rho_{0}\right)}=0$ and $x_{\left(\rho>\rho_{0}\right)}=-1$. As explained in the text and Ref. [54] the minimum radius is extended to, and kept at, $11.5 \mathrm{~km}$. The " 0 " characters (middle and right windows) denote values of $\Lambda_{1.4}$. See text for details.

the symmetry energy parameters $L, K_{\text {sym }}$, and the skewness $J_{\text {sym }}$ has been performed recently in Ref. [74]. Instead, in this work we investigate specifically the impact on $\Lambda$ of a transition from one density dependence of $E_{\text {sym }}$ to another (e.g., stiff-to-soft or soft-to-stiff), and assess whether precise measurements of the tidal deformability could distinguish such a transition.

The results of this analysis are displayed in Fig. 8, where we show the massradius relation (left panel) and $\Lambda$ as a function of the neutron-star mass $M$ (middle panel) and radius $R$ (left panel), and Table 2 which summarizes properties of $1.4 M_{\odot}$ neutron star models. We examine how the transition form stiff to soft and soft to stiff symmetry energy affects the tidal deformability. First, we start with the stiff-tosoft $E_{\text {sym }}$ transition. Specifically, we consider the case where for $x=-1(L=107$, $\left.K_{\text {sym }}=+95\right)$ for $\rho<\rho_{0}$ and $x=0\left(L=62, K_{\text {sym }}=-87\right)$ for $\rho>\rho_{0}$. We see that $R_{1.4}$ decreases from $13.6 \mathrm{~km}$ to $12.2 \mathrm{~km}, k_{2,1.4}$ from 0.0831 to 0.0661 and $\Lambda_{1.4}$ from 680 to 319, while compactness $\beta$ increases from 0.15 to 0.17 . Similarly, for the case of $x_{\left(\rho<\rho_{0}\right)}=-1$ and $x_{\left(\rho>\rho_{0}\right)}=0.3 R_{1.4}$ decreases to $11.6 \mathrm{~km}, k_{2,1.4}$ to 0.0537 and $\Lambda_{1.4}$ to 161 , while $\beta$ increases to 0.19 . More generally, such qualitative behavior is observed in all instances 
Table 2. Properties of $1.4 M_{\odot}$ neutron star (NS) models with the MDI EOS with varied $E_{\text {sym }}(\rho)$ as discussed in the text. NS properties are given for three representative values of $x$ for $\rho<\rho_{0}$ : $-1,0$, and +1 . For each $x$ below $\rho_{0}$, the parameters $L$ and $K_{\text {sym }}$ are also included. The first column indicates the value of the parameter $x$ below saturation density. The remaining columns exhibit the following quantities: $x$ for $\rho>\rho_{0}$; compactness parameter $\beta$; NS radius $R(\mathrm{~km})$; Love number $k_{2}$; dimensionless tidal deformability $\Lambda$.

\begin{tabular}{c|ccccc}
\hline$x_{\left(\rho<\rho_{0}\right)}$ & $x_{\left(\rho>\rho_{0}\right)}$ & $\beta$ & $R$ & $k_{2}$ & $\Lambda$ \\
\hline-1 & -2 & 0.14 & 14.5 & 0.0960 & 1074 \\
$L=107$ & -1 & 0.15 & 13.6 & 0.0831 & 680 \\
$K_{\text {sym }}=95$ & 0 & 0.17 & 12.2 & 0.0661 & 319 \\
& 0.3 & 0.19 & 11.1 & 0.0537 & 161 \\
\hline 0 & -2 & 0.15 & 13.8 & 0.0980 & 876 \\
$L=62$ & -1 & 0.16 & 13.2 & 0.0882 & 615 \\
$K_{\text {sym }}=-87$ & 0 & 0.17 & 11.9 & 0.0707 & 292 \\
& 0.3 & 0.19 & 10.8 & 0.0571 & 149 \\
\hline+1 & -2 & 0.15 & 13.5 & 0.1032 & 811 \\
$L=48$ & -1 & 0.16 & 12.8 & 0.0937 & 577 \\
$K_{\text {sym }}=-142$ & 0 & 0.18 & 11.7 & 0.0756 & 287 \\
& 0.3 & 0.19 & 10.6 & 0.0607 & 145 \\
\hline
\end{tabular}

where $x_{\left(\rho<\rho_{0}\right)}<x_{\left(\rho>\rho_{0}\right)}$. Next, we look at the soft-to-stiff $E_{s y m}(\rho)$ transition, where as a representative example we consider the case of $x_{\left(\rho<\rho_{0}\right)}=0$ and $x_{\left(\rho>\rho_{0}\right)}=-1$. We observe that $R_{1.4}$ increases from $11.9 \mathrm{~km}$ to $13.2 \mathrm{~km}, k_{2,1.4}$ from 0.0707 to 0.0882 , and $\Lambda_{1.4}$ from 292 to 615 , while $\beta$ decreases from 0.17 to 0.16 . This trend is seen in all cases where $x_{\left(\rho<\rho_{0}\right)}>x_{\left(\rho>\rho_{0}\right)}$. For the stiff-to-soft $E_{s y m}$ transition we also notice that although $R, k_{2}$ and $\Lambda$ shift toward their canonical values (as summarized in Table 1), the actual decreased values are always larger than the canonical ones. Conversely, for the soft-to-stiff $E_{\text {sym }}$ transition, the increased values of $R, k_{2}$, and $\Lambda$ tend to be smaller than their canonical counterparts. The observed behavior is easily understood, and best interpreted, in terms of the modified total pressure (left panel of Fig. 7). For instance, in the case of a stiff-to-soft $E_{\text {sym }}$ transition the dips exhibited by the $x=0$ and $x=0.3$ curves (in the upper left window of Fig. 7) indicate a decrease in $P$, as compared to the $x=-1$ curve for $\rho<\rho_{0}$, and result in overall softer EOSs and more centrally condensed neutron-star models with smaller $R$ and $\Lambda$. By the same token, in the case of a softto-stiff $E_{\text {sym }}$ transition, the humps exhibited by the $x=-1$ and $x=-2$ curves (in the middle left window of Fig. 7) represent an increase in $P$, as compared to the $x=0$ curve for $\rho<\rho_{0}$, and lead to less compact NS configurations with larger $R$ and $\Lambda$. In addition, the corresponding decreased (increased) values of $R$ and $\Lambda$ are larger (smaller) than their canonical counterparts because the resultant modified EOSs used to compute the NS properties are overall stiffer (softer) than the unaltered EOSs (computed with the same $x$ for the whole density range). 
It is also seen in Fig. 8 that as $x_{\left(\rho<\rho_{0}\right)}$ increases from -1 (upper frames) to 0 (middle frames), to +1 (lower frames), $R$ and $\Lambda$ decrease and this effect is best observed in the left panel. It is signified by the diminishing area of the uncolored rectangular region defined by $R_{1.4}$ and $\Lambda_{1.4}$ computed with the MDI EOS with $x=-1$ and $x=0$ above $\rho_{0}$. This behavior is attributed to the overall softening of the EOS due to the increase of $x$ below saturation density. Most importantly, it is also observed that the region representing the MDI EOS results (uncolored rectangle) overlaps significantly with the colored rectangular region denoting the gravitational wave constraints, with the overlap being complete in the lower left frame of Fig. 8. These findings suggests that even if such a low-density phase transition exists, it would not be visible in the current GW170817 data. They also illustrate that a variety of density behaviors of the symmetry energy, as parameterized here by $x_{\left(\rho<\rho_{0}\right)}$ and $x_{\left(\rho>\rho_{0}\right)}$, could yield the same $\Lambda$. More qualitatively, since a single $x$ value corresponds to specific values of $L$ and

$K_{\text {sym }}$ (Fig. 2), varying $x$ below and above saturation density is equivalent to varying the slope and curvature of $E_{\text {sym }}$, and many different combinations of large (small) slope and small/negative (large/positive) curvature lead to the same $\Lambda_{1.4}$. In this respect, a precise measurement of $\Lambda_{1.4}$ restricts the configuration space of possible $L$ and $K_{\text {sym }}$, but not the individual parameters of $E_{\text {sym }}$ themselves. These conclusions are consistent with recent investigations [74] where the effects on $\Lambda_{1.4}$ of varying $L, K_{\text {sym }}$ and $J_{\text {sym }}$ are studied systematically with a parameterized EOS. There does not appear to exist a simple relationship between the tidal deformability and the slope of the symmetry energy (hence the neutron-star radius $R$ ) alone, and the reported relations are rather model dependent [80]. As already pointed out in the literature, translating $\Lambda$ measurements directly into $R$ constraints has to be taken with some caution [80]. Therefore, both $R$ and $\Lambda$ have to be measured independently to pin down the details of $E_{\text {sym }}(\rho)$.

It is expected, in the near future, that many more observations of GWs from merging neutron star binaries with the advanced ground based and scheduled spacebourne gravitational-wave detectors will become available, and allow for precise $\Lambda$ measurements. Such tighter constraints on the tidal deformability together with precise measurements of the neutron-star radius from X-ray observations will determine the exact correlation between $R$ and $\Lambda$ and help further understand the high-density $E_{\text {sym }}$ and EOS.

\section{Summary and outlook}

In summary, using the MDI EOS which has its SNM part and the low-density symmetry energy $E_{\text {sym }}(\rho)$ constrained by earlier data from heavy-ion reactions, we have investigated the effect of the symmetry energy $E_{\text {sym }}(\rho)$ on the tidal properties of coalescing binary neutron stars. For $1.4 M_{\odot}$ neutron-star models, the dimensionless tidal deformability $\Lambda_{1.4}$ is found to be in the range of $\sim[292-680]$. These estimates based on nuclear laboratory data are in excellent agreement with the constraints on $\Lambda_{1.4}$ from the GW170817 event [3]. The GW170817 event provides a limit for the radius of canonical neutron stars. It is consistent with but less restrictive than the earlier prediction based 
on the EOS partially constrained by the experimental data of heavy-ion reactions. We also investigated the effect of varying $E_{\text {sym }}(\rho)$, below and above $\rho_{0}$, on tidal properties of neutron stars and found that while $\Lambda_{1.4}$ is rather sensitive to this variation, many different combinations of large (small) $E_{\text {sym }}$ slope and small/negative (large/positive)

$E_{\text {sym }}$ curvature yield the same $\Lambda_{1.4}$. Specifically, we studied the transition from stiff to soft $E_{\text {sym }}$ at $\rho_{0}$ and our findings suggest that even if such a low-density transition exists it would not be visible in the current GW170817 data. Therefore, additional probes including ones from terrestrial nuclear laboratories are required resolve this degeneracy and determine the precise density behavior of $E_{\text {sym }}$. In this respect, the results in this work signify the importance of coherent analyses of dense neutron-rich nuclear matter EOS underlying both nuclear laboratory experiments and astrophysical observations.

The gravitational wave astronomy is at its very beginning but it has already started providing important insights about the nature of compact stars and the EOS of dense neutron-rich matter. In the near future, besides the ongoing and planned X-ray observations of neutron stars, more neutron star merger events, together with a new generation of gravitational-wave detectors and refined waveform models, will certainly help us better understand the structure of compact stars and the nature of dense neutron-rich matter. Similarly, new experiments in terrestrial nuclear laboratories, especially at advanced rare isotope beam facilities, will also provide greater details about the EOS of neutron-rich matter, in particular about the high-density behavior of the nuclear symmetry energy. A truly multi-messenger approach combining results from both terrestrial laboratories and astrophysical observations will finally allow us to pin down the EOS of neutron-rich matter in a broad density range.

\section{Acknowledgments}

We would like to thank F. J. Fattoyev, N.B. Zhang and J. Xu for helpful communications and discussions. This work is supported in part by the U.S. Department of Energy, Office of Science, under Award Number de-sc0013702, the CUSTIPEN (China-U.S. Theory Institute for Physics with Exotic Nuclei) under the US Department of Energy Grant No. de-sc0009971, the National Natural Science Foundation of China under Grant No. 11320101004 and the Texas Advanced Computing Center.

\section{References}

[1] Abbott B P et al. (Virgo, LIGO Scientific) 2017 Phys. Rev. Lett. 119161101

[2] Abbott B P et al. (Virgo, LIGO Scientific) 2017 Astrophys. J. $848 \mathrm{~L} 12$

[3] Abbott B P et al. (Virgo, LIGO Scientific) 2018 Phys. Rev. Lett. 121161101

[4] Radice D, Perego A, Zappa F and Bernuzzi S 2018 Astrophys. J. 852 L29

[5] Hinderer T, Lackey B D, Lang R N and Read J S 2010 Phys. Rev. D 81123016

[6] Flanagan E E and Hinderer T 2008 Phys. Rev. D 77021502

[7] Shibata M 2015 Numerical Relativity (World Scientific Publishing Co. Pte. Ltd.)

[8] Kochanek C S 1992 Astrophys. J. 398234

[9] Bildsten L and Cutler C 1992 Astrophys. J. 400175

[10] Lai D and Wiseman A G 1996 Phys. Rev. D $\mathbf{5 4} 3958$

[11] Aasi J et al. (Virgo, LIGO Scientific) 2015 Class. Quant. Grav. 32115012 
[12] Acernese F et al. (VIRGO Collaboration) 2015 Class. Quant. Grav. 32024001

[13] Aso Y et al. (KAGRA Collaboration) 2013 Phys. Rev. D 88043007

[14] Dominik M et al. 2015 Astrophys. J. 806263

[15] Haas R et al. 2016 Phys. Rev. D 93124062

[16] Hinderer T et al. 2016 Phys. Rev. Lett. 116181101

[17] Vidana I, Providencia C, Polls A and Rios A 2009 Phys. Rev. C 80045806

[18] Danielewicz P, Lacey R and Lynch W G 2002 Science 2981592

[19] Li B A, Ko C M and Bauer W 1998 Int. J. Mod. Phys. E 7147

[20] Eds. Li B A and Schröder W U 2001 Isospin Physics in Heavy-Ion Collisions at Intermediate Energies (Nova Science Publishers, Inc, New York)

[21] Lattimer J M and Prakash M 2001 Astrophys. J. 550426

[22] Lattimer J M and Prakash M 2004 Science 304536

[23] Baran V, Colonna M, Greco V, and Toro M D 2005 Phys. Rep. 410335

[24] Steiner A W, Prakash M, Lattimer J M and Ellis P J 2005 Phys. Rep. 411325

[25] "Topical issue on nuclear symmetry energy" Eds. Li B A, Ramos A, Verde G and Vidaña I 2014 Eur. Phys. J. A $\mathbf{5 0}$ No. 2

[26] Balantekin A B, Carlson J, Dean D J, Fuller G M, Furnstahl R J, Hjorth-Jensen M, Janssens R V F, Li B A, Nazarewicz W, Nunes F M, Ormand W E, Reddy S and Sherrill B M 2014 Modern Physics Letters A 291430010

[27] Li B A, Chen L W and Ko C M 2008 Phys. Rep. 464113

[28] Tsang B M et al. 2012 Phys. Rev. C 86105803

[29] Li B A and Han X 2013 Phys. Lett. B727 276

[30] Horowitz C J, Brown E F, Kim Y, Lynch W G, Michaels R, Ono A, Piekarewicz J, Tsang M B, Wolter H 2014 J. Phys. G: Nucl. Part. Phys. 41093001

[31] Lattimer J M and Steiner A W 2014 European Phys. Journal A 50 40; 2014 Astrophys. J 784 123

[32] Baldo M and Burgio G F 2016 Prog. Part. Nucl. Phys. 91203

[33] Hinderer T 2008 Astrophys. J. 6771216

[34] Binnington T and Poisson E 2009 Phys. Rev. D 80084018

[35] Damour T and Nagar A 2009 Phys. Rev. D 80084035

[36] Postnikov S, Prakash M and Lattimer J M 2010 Phys. Rev. D 82024016

[37] Moustakidis C C, Gaitanos T, Margaritis C and Lalazissis G A 2017, Phys. Rev. C 95045801 ; Erratum: [2017 Phys. Rev. C 95 059904].

[38] Kumar B, Biswal S K and Patra S K 2017 Phys. Rev. C 95015801

[39] Zhou E P, Zhou X and Li A 2018 Phys. Rev. D 97083015

[40] Annala E, Gorda T, Kurkela A and Vuorinen A 2018 Phys. Rev. Lett. 120172703

[41] Fattoyev F J, Piekarewicz J and Horowitz C J 2018 Phys. Rev. Lett. 120172702

[42] Most E R, Weih L R, Rezzolla L and Schaffner-Bielich J 2018 Phys. Rev. Lett. 120261103

[43] Raithel C, zel F and Psaltis D 2018 Astrophys. J. 857 L23

[44] Tews I, Margueron J and Reddy S 2018 Phys. Rev. C 98045804

[45] Malik T, Alam N, Fortin M, Providncia C, Agrawal B K, Jha T K, Kumar B and Patra S K 2018 Phys. Rev. C 98035804

[46] Lim Y and Holt J W 2018 Phys. Rev. Lett. 121062701

[47] Fattoyev F J, Carvajal J, Newton W G and Li B A 2013 Phys. Rev. C 87015806

[48] Das C B, Gupta S D, Gale C and Li B A 2003 Phys. Rev. C 67034611

[49] Li B A, Das C B, Gupta S D and Gale C 2004 Phys. Rev. C 69011603 (R); 2004 Nucl. Phys. A 735563

[50] Chen L W, Ko C M and Li B A 2005 Phys. Rev. Lett. 94 032701; Li B A and Chen L W 2005 Phys. Rev. C $\mathbf{7 2} 064611$

[51] Xu J, Chen L W, Li B A and Ma H R 2007 Phys. Lett. B650 348

[52] Constantinou C, Muccioli B, Prakash M and Lattimer J M 2015 Phys. Rev. C 92 025801; ibid 2015 Annals Phys. 363533

[53] Tsang M B et al. 2004 Phys. Rev. Lett. 92062701 
[54] Steiner A W and Li B A 2005 Physical Review C 72 041601; Li B A and Steiner A W 2006 Phys. Lett. B 642436

[55] Akmal A, Pandharipande V R and Ravenhall D G 1998 Phys. Rev. C 581804

[56] Alonso D and Sammarruca F 2003 Phys. Rev. C 67054301

[57] Krastev P G and Sammarruca F 2006 Phys. Rev. C 74025808

[58] Machleidt R 1989 Adv. Nucl. Phys. 19189

[59] Pandharipande V R and Ravenhall D G 1989 Hot Nuclear Matter in Nuclear Matter and Heavy Ion Collisions (NATO ADS Ser.) vol B205 ed. Soyeur M, Flocard H, Tamain B and Porneuf M (Dordrecht: Reidel) p 103

[60] Douchin F and Haensel P 2001 Astron. Astrophys. 380151

[61] Pethick C J, Ravenhall D G and Lorenz C P 1995 Nucl. Phys. A 584675

[62] Haensel P and Pichon B 1994 Astron. Astrophys. 283313

[63] Krastev P G, Li B A and Worley A 2008 Phys. Lett. B 6681

[64] Hessels J W T et al. 2006 Science 3111901

[65] Horowitz C J and Piekarewicz J 2001 Phys. Rev. Lett. 865647

[66] Krastev P G and Li B A 2007 Phys. Rev. C 76055804

[67] Jofre P, Reisenegger A and Fernandez R 2006 Phys. Rev. Lett. 97131102

[68] Krastev P G, Li B A and Worley A 2008 Astrophys. J. 6761170

[69] Worley A, Krastev P G and Li B A 2008 Astrophys. J. 685390

[70] Newton W G and Li B A 2009 Phys. Rev. C 80 065809; Newton W G, Gearheart M, Li B A 2012 The Astrophysical Journal Supplement Series 204 (1) 9

[71] Xu J, Chen L W, Li B A and Ma H R 2009 Astrophys. J 697 1549; Xu J, Chen L W, Ko C M and Li B A 2010 Phys. Rev. C $\mathbf{8 1} 055805$

[72] Friedman J L, Ipser J R and Parker L 1984 Nature 312255

[73] Zhang N B, Li B A and Xu J 2018 Astrophys. J. 85990

[74] Zhang N B and Li B A 2019 J. Phys. G 46014002

[75] Bhat S A and Bandyopadhyay D 2019 J. Phys. G 46014003

[76] Gandolfi S and Steiner A W 2016 J. Phys.: Conf. Ser. 665012063

[77] Steiner A W, Lattimer J M and Brown E F 2013 Astrophys. J. 765 L5

[78] Abrahamyan S et al. 2012 Phys. Rev. Lett. 108112502

[79] Horowitz C J et al. 2012 Phys. Rev. C 85032501

[80] Zhu Z Y, Zhou E P and Li A 2018 Astrophys. J. 86298 